\title{
Operant Conditioning of Head-Waving in Aplysia. II. Contingent Modification of Electromyographic Activity in Identified Muscles
}

\author{
David G. Cook and Thomas J. Carew \\ Departments of Psychology and Biology, Yale University, New Haven, Connecticut 06520
}

\begin{abstract}
Aplysia can readily exhibit operant conditioning of their headwaving response when bright light is used as aversive reinforcement (Cook and Carew, 1986). In the first paper of this series (Cook and Carew, 1989a), we showed that the electromyographic (EMG) activity of a discrete band of neck muscles, the lateral columellar muscles (LCMs) of Aplysia is significantly correlated with the component of head-waving (the horizontal component) that is modified during operant conditioning. In the present paper, we asked whether the EMG activity of the LCMs themselves could also be contingently modified, using the same procedures that produce operant conditioning of the behavioral response. Differential EMG from the LCMs was recorded in freely behaving animals with chronically implanted muscle cuff electrodes. Animals receiving aversive reinforcement (bright light) that was contingent upon specific patterns of LCM activity readily learned to alter their differential EMG output. Like operant conditioning of the head-waving response, this operant modification of LCM activity was rapidly acquired and was specific to the contingencies of reinforcement.

These results show that a restricted group of muscles, the LCMs, exhibit the essential features of the head-waving system observed at the behavioral level: (1) their activity is significantly correlated with head-waving behavior, and (2) the LCMs are capable of operant modification of their output. Thus, this restricted response system provides a useful preparation for examining the neural mechanisms of operant conditioning of head-waving in Aplysia.
\end{abstract}

The head-waving response of Aplysia californica is a complex behavior that is used in a variety of behavioral contexts, including egg-laying (Cobbs and Pinsker, 1982a, b), feeding (Preston and Lee, 1973; Kupfermann, 1974; Bablanian et al., 1987), and locomotion (Hening et al., 1979; Fredman and Jahan-Parwar, 1983). We have found that this response can be operantly conditioned: animals can quickly learn to reduce head-waving to one side of their body to avoid presentation of an aversive bright light (Cook and Carew, 1986). Since Aplysia is well suited for a cellular analysis of behavior and behavioral plasticity $(\mathrm{Ca}-$

\footnotetext{
Received Dec. 8, 1988; revised Mar. 3, 1989; accepted Mar. 6, 1989.

We wish to thank Dorothy Shaffer and Russell Martin for their great assistance in collecting data, and Frederick Kuenzi and Mark Stopfer for their helpful criticism of the manuscript. This work was supported by NIH Grant 5-RO1-MH41083 and by NIH BRSG Grant 507-RR-075015 to T.J.C.

Correspondence should be addressed to Thomas J. Carew, Yale University, Departments of Psychology and Biology, Yale Station 11A, New Haven, CT 06520 .

Copyright (c) 1989 Society for Neuroscience $0270-6474 / 89 / 093107-08 \$ 02.00 / 0$
}

rew and Sahley, 1986; Byrne, 1987; Hawkins et al., 1987), the head-waving response of Aplysia provides an excellent system in which to examine the mechanisms of operant conditioning at a cellular level.

An important step in a cellular analysis of operant conditioning is to specify the motor pathways responsible for the operant response. The primary component of head-waving that is modified in our operant training procedure is the horizontal (side-to-side) component (Cook and Carew, 1986, 1989a). In the previous paper (Cook and Carew, 1989a), we identified a discrete, bilateral band of muscles in the neck and body wall, the lateral columellar muscles (LCMs) whose electromyographic (EMG) activity is significantly correlated with head-waving in the horizontal plane. In the present paper, we examine whether the EMG activity of the LCMs themselves can also be contingently modified using the same procedures that produce operant conditioning of the behavioral response. We found that the differential EMG activity of the LCMs can be significantly modified by the contingent presentation of aversive bright light. The operant modification of LCM activity, like the conditioning of the behavioral response, is rapidly acquired (within $10 \mathrm{~min}$ ) and is specific to contingent reinforcement since LCM activity in yoked control animals shows no operant modification. Therefore, this restricted muscle group in the head-waving system exhibits the essential features of operant conditioning of the complex behavioral response and thus provides a useful preparation in which to explore the cellular mechanisms of operant conditioning in Aplysia.

Some of the results described in this paper have been reported in abstract form (Cook and Carew, 1988).

\section{Materials and Methods}

Animals. Thirty adult $A$. californica $(150-250 \mathrm{gm})$ obtained from Sealife Supply (Sand City, CA) were used in the study. Animals were individually housed in an 1140 liter aquarium filled with circulating, aerated artificial seawater (Instant Ocean; Aquarium Systems, Mentor, $\mathrm{OH}$ ) maintained at room temperature $\left(20^{\circ}-22^{\circ} \mathrm{C}\right)$.

Muscle cuff electrodes. The EMG muscle cuff electrodes were constructed using the procedures of Pinsker and Eberly (1982) and were described in detail in the proceeding paper (Cook and Carew, 1989a). Briefly, the cuffs were made of $1-3 \mathrm{~mm}$ lengths of 0.025 inch i.d. Silastic tubing (Atlantic Healthcare Products; Andover, MA) that were split on one side lengthwise. Electrical contact with the muscle was made inside the cuff by means of a $1 \mathrm{~mm}$ length of bare $(0.001 \mathrm{inch})$ stress-relieved stainless steel wire (California Fine Wire; Grover City, CA). A $30 \mathrm{~cm}$ insulated elcctrode lead (made of the same wire) was coated with a thin layer of dental impression cement (Sybron/Kerr; Romulus, MI) and was led out of the animal to a preamplifier head-stage. The muscle cuff recordings were obtained with single-ended amplification.

Surgery. The implantation procedure is discussed in more detail in the preceding paper (Cook and Carew, 1989a). Briefly, prior to implantation the animals were anesthetized by injecting an amount of isotonic $\mathrm{MgCl}_{2}$ equal to $50 \%$ of body weight. Cuff electrodes were then bilaterally 
implanted through an incision on the lateral aspect of the neck on a band of the LCMs and sutured into position such that the LCM could contract and move normally. Following implantation, the incision was sutured closed and the animal was returned to its home tank, where it was allowed to recover for $24 \mathrm{hr}$ prior to training. Each animal was observed in the test chamber for at least 5-10 min prior to training to assure that normal head-waving behavior was exhibited and the EMG electrodes were functional. Similar applications of the cuff electrode technique (Pinsker and Eberly, 1982; Bablanian et al., 1987) appear to produce little or no impairment of muscle function (also see Cook and Carew, 1989a).

Apparatus. The training chamber was a $100 \times 100 \times 100 \mathrm{~cm}$ clear Plexiglas chamber enclosed in a Faraday cage. The animals were suspended underwater in the training chamber from small stainless steel hooks (inserted into the parapodia) attached to nylon tethers. In this position, animals could head-wave freely but could not touch the bottom or sides of the tank. The EMG electrode leads were wound around the suspension lines and then plugged into the preamplifier head-stages, which were located approximately $8 \mathrm{~cm}$ above the tank.

Bright light reinforcement ( 450 foot candles) was provided by 2 quartz halogen light sources (Dolan Jenner, Woburn, MA), delivered to the animal by 4 fiberoptic light guides positioned above each corner of the testing chamber and 2 incandescent lamps located $60 \mathrm{~cm}$ above the test chamber.

Recording apparatus. The EMG signals were amplified with Grass P511 A.C. preamplifiers (Quincy, MA). The amplified signals were recorded using an FM instrumentation recorder (Vetter, Rebersberg, PA). The muscle action potentials were counted by processing the signals through a voltage-level window discriminator (Frederick Haer, Brunswick, ME) that filtered out subthreshold noise and movement artifacts. The output of the window discriminators was monitored by a computer (IBM PC/AT and Data Translation 2821-F-8/DI data-acquisition system; Marlboro, MA) that counted, displayed, and stored each muscle action potential from both the left and right EMG channels.

Training and scoring procedures. The training protocol consisted of 3 phases: a $5 \mathrm{~min}$ baseline, a $10 \mathrm{~min}$ training period, and a $5 \mathrm{~min}$ test. Dim background illumination was provided by an incandescent lamp (1 foot candle), which remained illuminated throughout the baseline, training, and testing phases of the experiments. During training the computer monitored both EMG channels and determined the average spike rate for both the left and right LCM muscles in $1 \mathrm{sec}$ intervals. Furthermore, the computer calculated the average differential (left right) spike rate each second. Baseline scores were determined by summing the muscle action potentials that occurred on the left and the right sides during the baseline. Following the baseline period animals were randomly assigned to receive either contingent training or yoked control training. Contingently trained animals were "punished" by delivery of an aversive bright light if they exhibited increased activity in the muscle that showed the greater activity during the baseline compared with the LCM that had been less active. Each yoked control animal received noncontingent reinforcement with the identical pattern as a contingenttrained animal. In 12 of 15 cases each yoked control animal was yoked to a different contingent animal. Because animals were randomly assigned to treatment groups only after the completion of the baseline period, in 3 cases yoked controls were yoked to a contingent animal that had been used once before. Testing was performed in the absence of reinforcement. As in the baseline period, test scores were determined by summing the total number of muscle action potentials from the left and right LCMs.

Statistical analysis. Standard within-group and between-group comparisons were made by means of $t$-tests. Pilot experiments allowed us to predict the direction of all main effects. Thus, all probabilities are 1 -tailed.

\section{Results}

The primary goal of this study was to examine whether the electrical activity of the LCMs could be contingently modified by an operant training procedure known to produce operant conditioning of head-waving behavior in intact animals. If the differential electrical activity of the LCMs is capable of modification by operant training, 2 predictions follow. First, after contingent training, the differential output of the LCMs should exhibit contingent modification in the reinforced direction; and second, yoked control animals should show no net change in their LCM activity.

\section{Operant training procedure}

The preparation used to test the above predictions is shown in Figure 1. Twenty-four hours prior to an experiment, animals were surgically implanted with EMG cuff electrodes (Pinsker and Ebcrly, 1982; Cook and Carew, 1989a; see Materials and Methods). They were then suspended underwater in an operant conditioning chamber (Cook and Carew, 1986, 1989a), where ongoing EMG activity in the LCMs was continuously recorded. A schematic illustration of the training paradigm is illustrated in Figure 2. During a $5 \mathrm{~min}$ baseline, which was carried out in the absence of reinforcement, the average action potential frequency from both the left and right LCMs was determined. In previous behavioral experiments, we have observed that animals usually display a preference for head-waving to one side of the body (the preferred side is random from one animal to another). To illustrate this typical preference, in the schematic paradigm illustrated in Figure 2, in both the contingent and yoked control LCM pairs, the left LCM was arbitrarily chosen to be the least active of the pair during baseline.

For the animal that was to receive contingent training, the goal was to train the animal to change the differential firing ratc of the left and right LCMs in a fashion that would mimic the differential changes we observe during head-waving. This differential change could be accomplished by (1) increasing $(+)$ the firing rate of the less active LCM; (2) decreasing (-) the firing rate of the more active LCM; or (3) both increasing and decreasing the firing rates of the $(+)$ and $(-)$ LCMs, respectively. Onset and offset of reinforcement was delayed by approximately $2 \mathrm{sec}$ to insure that a particular EMG pattern was stable prior to delivery or termination of bright light. Thus, the contingencies of reinforcement would be delivered as shown in Figure 2. During the $10 \mathrm{~min}$ training period, negative reinforcement (bright light) is delivered when the left $(+)$ LCM fires more slowly than the right. The lights remain on until the right (-) LCM has reduced firing, the left $(+)$ has increased firing, or both. If animals are capable of modifying the output of the LCMs based upon contingent reinforcement, then during the $5 \mathrm{~min}$ test period, the left $(+)$ side should show an increase and the right $(-)$ side a decrease in EMG output compared with baseline. The yoked control animal illustrated in Figure 2 receives reinforcement that is identical to the contingent animal. However, since the reinforcement was not correlated with its own LCM activity, the yoked control animal should show no net shift in its EMG activity.

\section{Operant conditioning of the $L C M$}

Examples of the effects of contingent and yoked control training are provided in Figure 3, which shows sample EMG records (100 sec duration) from representative pairs of LCMs in a contingent-trained and in a yoked control animal. The sample traces are representative of activity during the 5 min baseline and test periods, and the 10 min training period.

During the baseline (Fig. $3 A_{l}$ ), the left LCM of the contingent animal was less active than the right; the left LCM was therefore designated as the $(+)$ muscle and the right LCM as the $(-)$ muscle. The EMG traces shown in the training phase (Fig. $3 A_{2}$ ) were taken 8 min after training had begun. Thus, the left LCM had already begun to show a substantial increase in EMG activity relative to the right $\mathrm{LCM}$. Towards the end of the trace 


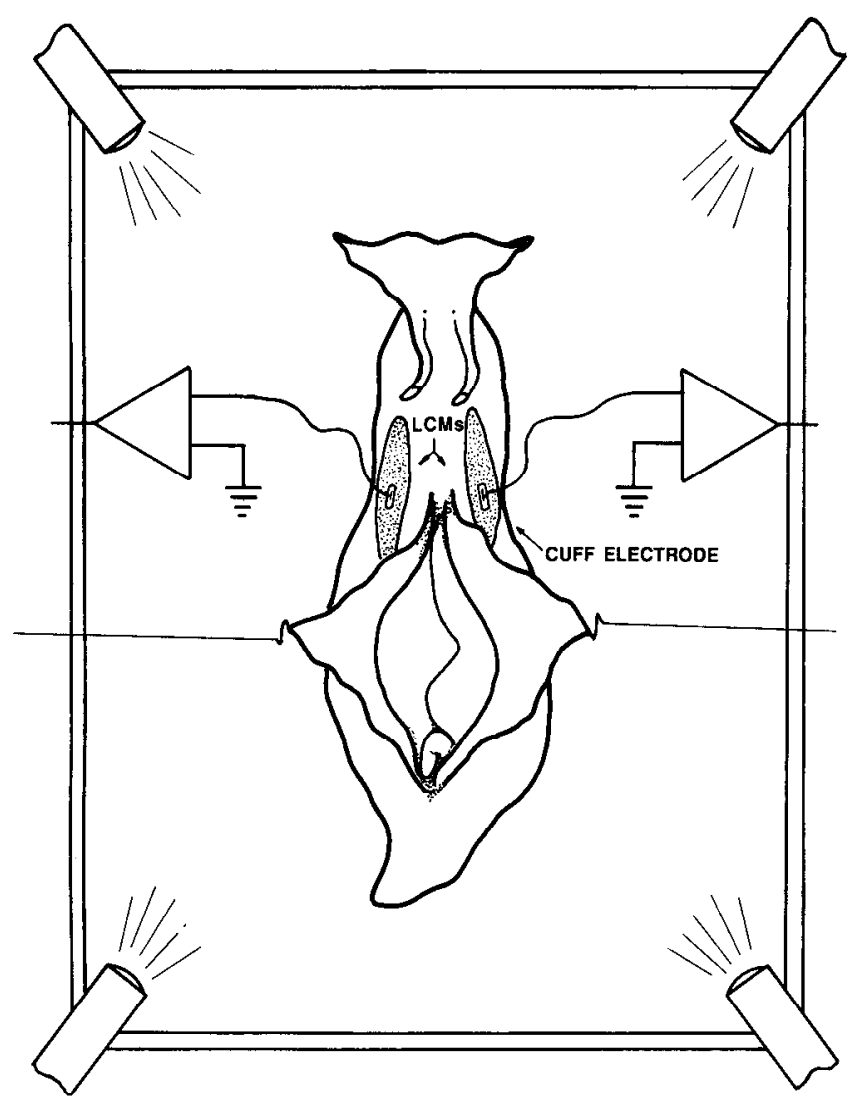

Figure 1. Experimental preparation. Animals were implanted with EMG cuff electrodes around bilateral pairs of LCMs. Twenty-four hours later, they were suspended underwater in an operant conditioning chamber (a dorsal view of the animal is depicted above). Bright lights in the 4 corners of the chamber serve as aversive reinforcement.

the lights were turned on because the differential firing rate of the 2 LCMs fell below the average differential baseline firing rate. The lights were again turned off when the left $\mathrm{LCM}$ resumed increased levels of activity. In the test phase (Fig. $3 A_{3}$ ), which was carricd out in the absence of reinforcement, the left $(+)$ LCM exhibited much greater EMG activity than during its own baseline, while the right $(-)$ LCM reduced its output compared with its baseline. The yoked control animal is shown in Figure
$3 B$. The EMG traces shown in this figure are from the animal whose reinforcement was yoked to the reinforcement of the contingent animal in Figure $3 A$. Baseline rates for the 2 animals were comparable. Records from the training period (Fig. $3 B_{2}$ ) show that reinforcement was not correlated with the activity of the LCM pair in the yoked control animal (notice that the lights were turned on during a period in which the LCMs showed very little change in their firing pattern). Finally, during the test period (Fig. $3 B_{3}$ ), the yoked control LCM pair showed no clear change in their differential firing pattern compared with baseline. Thus, for the 2 animals shown in Figure 3, it is evident that the LCMs of the animal receiving contingent training showed a shift in differential EMG activity that is consistent with the animal learning to turn the lights off and keep them off. The yoked control animal, on the other hand, showed no evidence of learning anything about the light.

The results shown in Figure 3 provide sample activity during baseline, training, and test periods. In Figure 4, EMG activity throughout the entire baseline and test periods for these same 2 animals is shown. In these graphs, the average differential spike rate (left EMG - right EMG) is shown as a function of time for both the contingent animal (Fig. $4 A$ ) and yoked control animal (Fig. $4 B$ ). Data points above zero (horizontal dashed line) indicate that the left LCM was more active than the right; conversely, data points below zero indicate that the right LCM was more active than the left. For the contingent animal shown in Figure $4 A_{l}$, throughout most of the baseline, its right LCM had a higher rate than the left. However, during the test, the pattern was reversed. For nearly the entire test period the firing rate of the left LCM exceeded the right. These results are in contrast to the LCMs from the yoked control animal (Fig. $4 B_{l}$ ), which showed no systematic change in firing pattern following training: Early in the test period, the left LCM was relatively more active than the right, but this pattern reversed in the second half of the test period, with the result being no net change in differential LCM firing rate compared with baseline.

The summed averages of the differential spikes rates during the baseline and test periods for the contingent and yoked control LCM pairs are shown in Figures $4 A_{2}$ and $4 B_{2}$. Data above the abscissa indicate that, on average, the left LCM showed more activity than the right LCM; conversely, data below the abscissa indicate that the right was more active than the left. The contingent LCM pair is shown in Figure $4 A_{2}$. During the

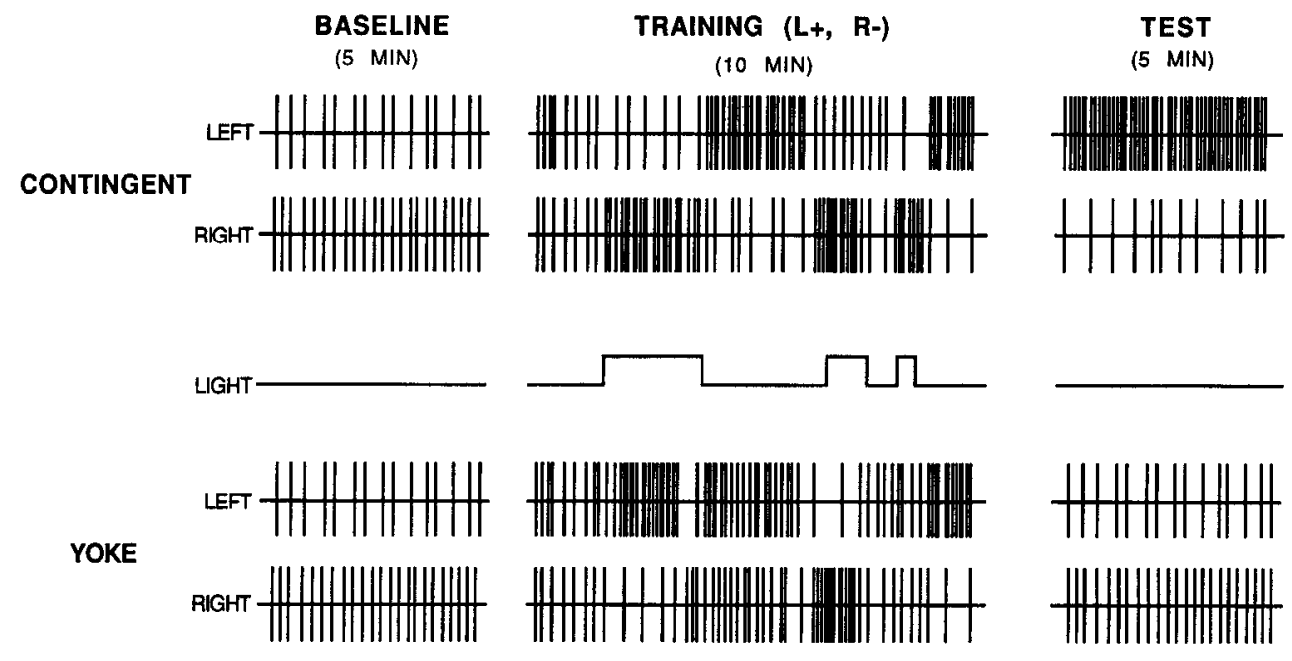

Figure 2. Experimental paradigm for contingent modification of LCM activity. Each pair of traces is a schematic illustration of EMG activity in left and right LCMs, respectively. Contingenttrained and yoked control animals are treated identically in the baseline and test periods (carried out in dim illumination). During training, contingent animals are conditioned to change the differential firing rate of their left and right LCMs. Thus, they receive aversive reinforcement when the LCM that was less active in baseline (in this example, the left LCM) remains low with respect to the more active LCM. Yoked control muscles received identical reinforcement, but it was not contingent upon the yoked LCM activity. 


\section{CONTINGENT}

$A_{1}$
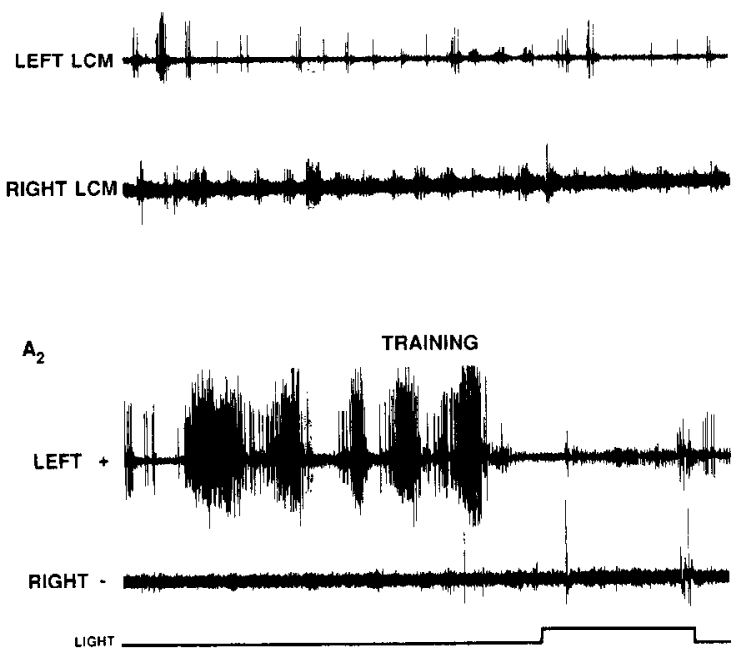

$A_{3}$

LEFT



$\mathbf{B}_{1}$

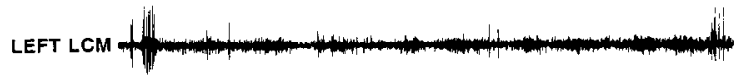

$\mathrm{B}_{2}$

LEFT

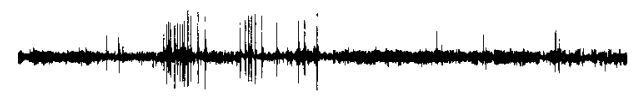

RIGHT



$\mathbf{B}_{3}$

LEFT

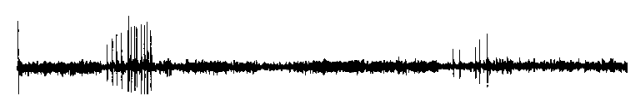

RIGHT

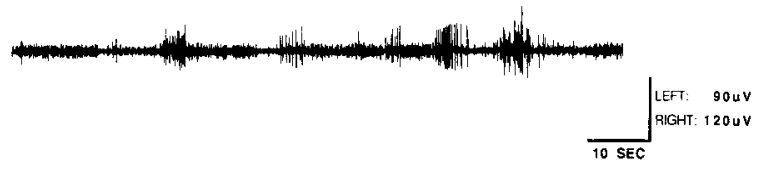

Figure 3. Examples of contingent and yoked control LCM activity. $A$, Contingent-trained LCMs. Each pair of traces comprises EMG recordings $(100 \mathrm{sec})$ from left and right LCMs, respectively, in frecly behaving animals. $A_{t}$, During basclinc, the left LCM is less active than the right; thus, it is chosen to be the $(+)$ muscle. $A_{2}$, Following $8 \mathrm{~min}$ of contingent training, the left LCM is substantially more active, and the right less active; thus, no reinforcement occurs (lights are off). Towards the end of the trace, the left LCM fell below the right, which caused the lights to come on until the left once again exceeded the right (at end of the record). $A_{3}$, During testing, the left $(+)$ LCM shows greater EMG activity compared with its own baseline, while the right LCM decreased its activity compared with baseline. $B$, Yoked control LCMs. $B_{l}$, Baseline EMG activity is comparable to the contingent-trained animal $A_{1} . B_{2}$, The same pattern of reinforcement as delivered in the training phase of $A_{2}$ is delivered to this animal but was uncorrelated with its LCM activity. $B_{3}$, During the test phase, there was no substantial change in LCM activity compared with the baseline in $B_{l}$.

baseline, the right LCM fired more rapidly on average than the left LCM. Following training, during which the animal was punished for producing differential spike rates below zero (i.e., right $>$ left) the LCM pair showed a highly significant increase in differential rates above zero (left $>$ right) $(t[298]=2.68, p<$ $0.007)$. The yoked control LCM pair is shown in Figure $4 B_{2}$. During the baseline, the yoked control LCMs showed roughly equivalent levels of activity as the contingent LCMs above. Following training, the yoked LCM pair showed no significant differential change in LCM activity $(t[298]=0.61$, n.s. $)$.

Thus far, we have shown a representative cxamplc of a contingent-trained and a yoked control LCM pair. In this study, 30 animals were trained (15 contingent, 15 yoked control). A summary of all of the data is shown in Figure 5, which illustrates the net contingent modification exhibited by contingent-trained LCM pairs, as well as the changes exhibited by yoked control LCM pairs. Net change in EMG has been quantified as a difference score between the average differential EMG spike rate during the test period and the baseline period (see Materials and
Methods). A within-group analysis revealed that, following training, contingent-trained LCM pairs showed a significant increase in differential LCM activity in the reinforced direction (average change in differential spike rate $=3.25, \pm 1.35 ; t[14]$ $=2.4, p<0.025$ ). Yoked controls, on the other hand, showed no significant change in differential LCM activity (average change in differential spike rate $=0.16, \pm 0.49 ; t[14]=0.32$, n.s.). Moreover, a between-group comparison revealed that the contingent-trained animals showed a significantly greater change in differential EMG than did the yoked controls $(t[28]=2.15, p$ $<0.025$ ). Prior to training, there were no significant differences between the contingent and yoked control LCMs in either the $(+)$ or $(-)$ baseline scores.

Given that contingent reinforcement produced significant modification of EMG from the LCMs (Fig. 5), it was of interest to determine whether, in the same animals, the head-waving response was also contingently modified. To examine this question, we analyzed the behavioral learning scores of the animals following training of the LCMs. As in previous behavioral ex- 


\section{CONTINGENT}

$A_{1}$

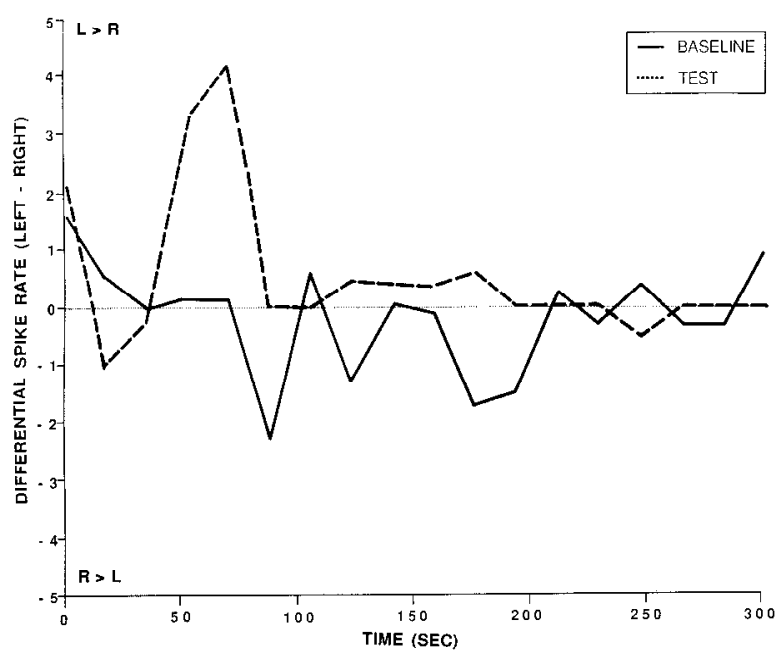

YOKED CONTROL

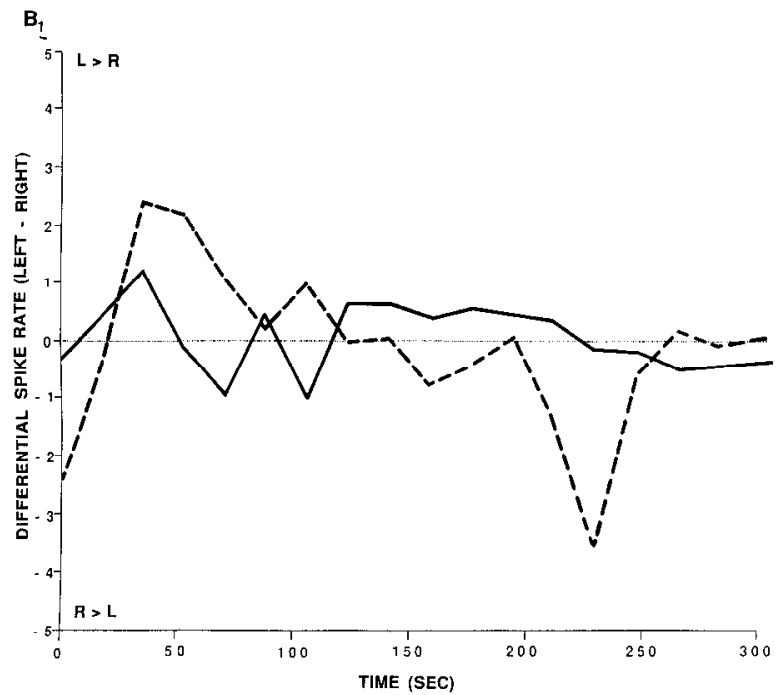

$A_{2}$

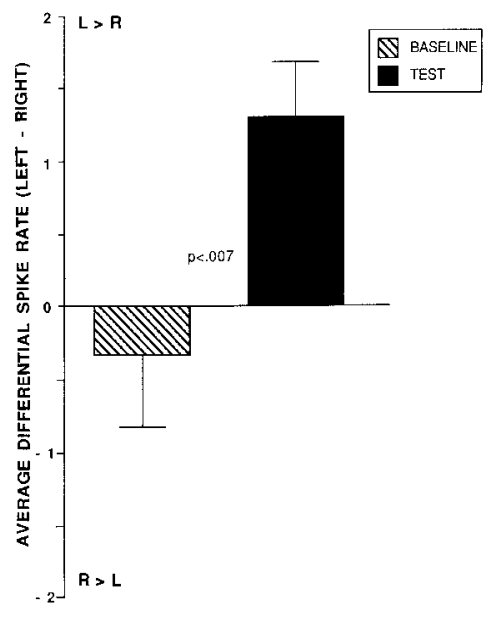

$\mathrm{B}_{2}$

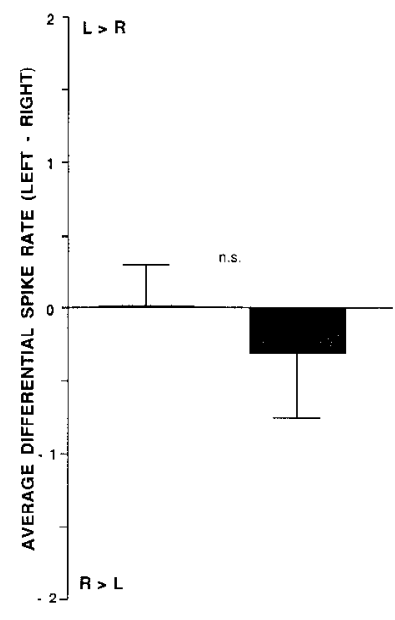

Figure 4. Contingent modification of LCM activity. Data are summaries of entire baseline and test periods $(300 \mathrm{sec})$ for the animals shown in Figure 3. Data are expressed as differential (left - right) LCM spike rate. The horizontal dashed line through the zero intercept indicates no difference in rates; data points above the dashed line indicate that left LCM activity exceeded the right, and below the line that right LCM activity exceeded the left. $A_{l}$, Throughout most of the baseline period (solid line), the right LCM activity excecded the left. However, during the test (dotted line), the pattern was reversed. $A_{2}$, Statistical analysis of data shown in $A_{l}$. The average differential spike rate of the LCMs is significantly altered by contingent reinforcement $(p<0.007) . B_{\text {, }}$, There was no systematic change in baseline LCM activity (solid line) compared with test activity (dotted line). $B_{2}$, There was no significant modification of LCM activity in this animal, whose reinforcement was yoked to the contingent-trained animal shown in $A$. periments (Cook and Carew, 1986), operant conditioning was assessed by comparing the amount of time (in seconds) spent head-waving on the "safe" (unpunished side) during the test pcriod relative to the baseline period (test - baseline). Interestingly, although the LCMs clearly exhibited a significant change in activity following training, the head-waving response did not show significant modification [contingent-trained $(n=10)=$ $-15.8 \pm 40.5$; yoked controls $(n=8)=-14.5 \pm 25.33$; n.s.]. These results suggest 2 conclusions: (1) In parallel with the LCMs, other muscle systems may be required to produce operant conditioning of the complex behavioral response; and (2) the activity of the LCMs may actually provide a more sensitive assay of contingent modification than the intact behavioral response (see Discussion).

\section{Discussion}

Operant conditioning in Aplysia

Learning can be divided into 2 broad categories: nonassociative and associative. Associative learning, in turn, can be divided into 2 principal classes: Pavlovian (classical conditioning) and instrumental (operant) conditioning (Mackintosh, 1974). In Pavlovian conditioning, an animal learns to associate 2 stimuli: a conditioned stimulus (CS), which scrves as a signal or predictor, and an unconditioned stimulus (US), which serves as a reinforcer (Rescorla, 1988). In contrast, in operant conditioning an animal learns to associate a behavioral response (the operant response) with the occurrence of reinforcement. Positive (rewarding) reinforcement typically increases the probability of a response, whereas punishing reinforcement usually reduces the probability of responding (Reynolds, 1968). In recent years, considerable progress has been made toward achieving a cellular and molecular understanding of classical conditioning. For example, significant advances have recently been made in 2 invertebrate preparations: Hermissenda (Farley and Alkon, 1982; Crow, 1985a, b; Alkon, 1987; Crow, 1988) and Aplysia (Carew et al., 1983; Hawkins et al., 1983; Walters and Byrne, 1983a, b). Operant conditioning, on the other hand, is poorly understood in cellular terms. Thus, we were interested in determining 


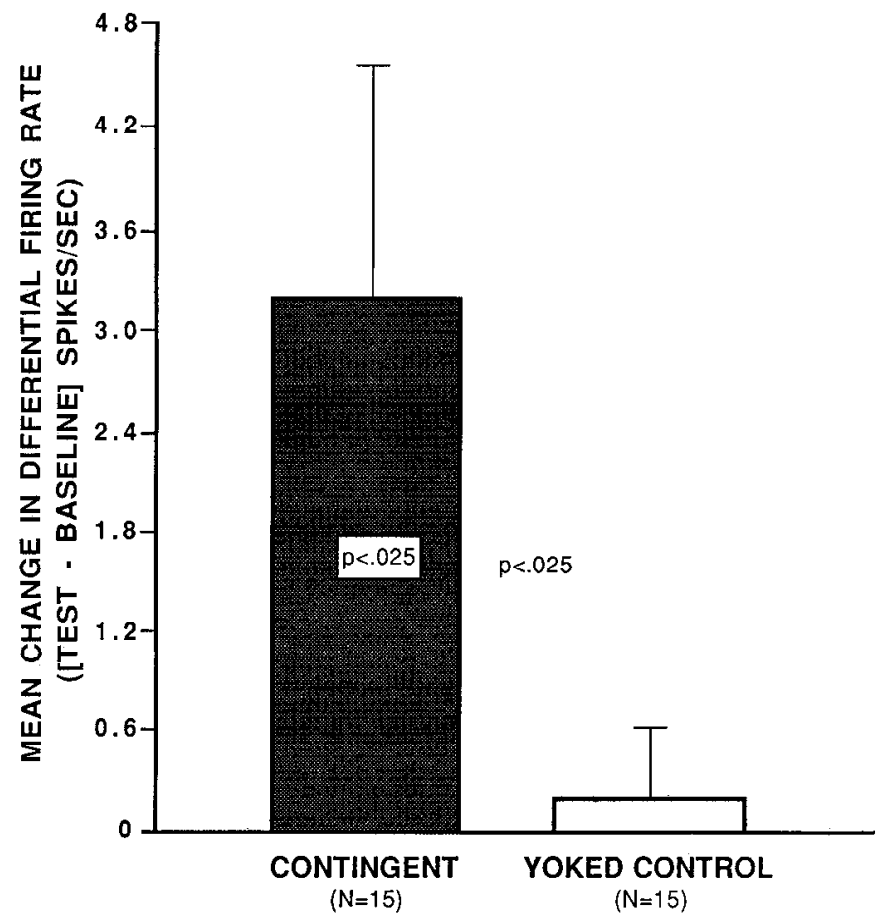

Figure 5. Summary of all experiments. Data are expressed as a difference score between cumulative differential EMG activity during the test and baseline periods. Contingent-trained LCM pairs $(n=15)$ showed a significant increase in the reinforced direction $(p<0.025)$, whereas yoked controls $(n=15)$ showed no within-group change. Moreover, following training, contingent-trained LCM pairs showed a significantly greater change in differential firing rate than yoked controls $(p<0.025)$.

whether Aplysia could also exhibit operant conditioning since this animal has proven to be well suited for a cellular and molecular analysis of a variety of forms of learning.

The behavioral response that we have examined is the headwaving responsc of Aplysia. Hcad-waving is a naturally occurring behavior in which Aplysia sweep their heads from side to side in order to explore their environment, for example, to locate food or find a substrate on which they can attach their foot (Kupfermann and Carew, 1974; Cook and Carew, 1989a). We have found that when animals are suspended under water but above a substrate, they will head-wave vigorously for up to an hour. This response can be operantly conditioned using aversive bright light as reinforcement. Specifically, if head-waving to one side of the body (the punished side) results in aversive reinforcement, and head-waving to the opposite side (the safe side) results in the termination of that reinforcement, animals will learn to reduce head-waving on the punished side and increase it on the safe side.

A key feature of operant conditioning in higher animals is that the learning is sensitive to the contingencies of reinforcement (Reynolds, 1968; Mackintosh, 1974). Operant conditioning of the head-waving response in Aplysia is clearly influenced by the contingencies of reinforcement, as revealed by 2 kinds of experiments. (1) If animals are initially trained to increase head-waving to a safe side (for example, the left side) and then the contingencies of a reinforcement are reversed (the left side now becoming the punished side), animals show a significant decrease in head-waving to the newly punished side. However, when the original reinforcement contingencies are reinstated, animals once again show a significant increase in head-waving to the safe (left) side. (2) Yoked control animals, which receive uncorrelated reinforcement, show no learning. However, when yoked animals are subsequently exposed to contingent reinforcement, they readily acquired the operant learning. Thus, similar to operant conditioning in higher animals, operant conditioning of head-waving in Aplysia exhibits clear sensitivity to variations in the contingencies of reinforcement (Cook and $\mathrm{Ca}$ rew, 1986).

The capacity for operant conditioning is not confined to the head-waving response of Aplysia. There was the suggestion in early studies by Lee $(1969,1970)$ that Aplysia could learn to occupy a certain position in an aquarium in order to obtain an increase in water level. Subsequently, Downey and Jahan-Parwar (1972) suggested that the critical rcinforcement in the studies by Lee was not water level but water temperature. Although interesting, these initial studies have not been pursued further in Aplysia. More recently, an operant-like modification of feeding behavior has been reported by Susswein and Schwarz (1983), who found that, if Aplysia are presented with inedible food (by putting it in a plastic net), they will gradually stop responding to that food but not to other foods. Subsequent experiments have revealed several important features of this form of learning (Schwarz and Susswein, 1986; Susswein et al., 1986), which are promising both from a behavioral perspective and for further neurophysiological studies (see, for example, Susswein and Byrne, 1988). Finally, operant conditioning of gill withdrawal has been reported by Hawkins et al. (1985), who found that Aplysia can learn to keep their gills contracted to a specified criterion level to avoid presentation of shock to the siphon. Taken collectively these results show that Aplysia may be capable of expressing operant conditioning in a number of different response systems.

In addition to experiments done in Aplysia, operant conditioning has received attention in other invertebrate animals, such as conditioned leg lifting in the grasshopper (Woolacott and Hoyle, 1977; Hoyle, 1982a, b) and operant punishment of eye elevation in the green crab (Abramson and Feinman, 1987). Morcover, in a mammalian system, the rat hippocampal slice preparation, Stein and Belluzzi (1988) have recently reported that individual neurons can undergo operant conditioning using punctate injections of dopamine as the reinforcement. Thus, it may soon be possible to compare the cellular mechanisms that produce operant conditioning in different response systems of Aplysia, in other invertebrates, and in the mammalian brain. Such comparisons will certainly contribute to our overall understanding of instrumental learning, as well as its relationship to classical conditioning.

\section{Operant conditioning of the LCMS}

Head-waving in Aplysia is mediated by a complex array of longitudinal, transverse, and oblique muscles in the neck and head (Brace, 1977a, b; Bablanian et al., 1987; Kuenzi and Carew, 1988). Among these muscles the LCMs are anatomically well situated to participate in horizontal head-waving. In the previous paper, we showed that differential EMG activity in the LCMs is significantly correlated with horizontal head-waving behavior (Cook and Carew, 1989a). In the present study we have shown that the differential activity of the LCMs can be contingently modified using the same reinforcement and training procedures that induce behavioral operant conditioning of head-waving (Cook and Carew, 1986). Specifically, animals that received contingent reinforcement of differential LCM activity 
showed a significant increase in the differential firing rate of the LCMs in the reinforced direction compared with their own baseline activity. In contrast, yoked control animals, which received an identical pattern of reinforcement except that the reinforcement was uncorrelated with their LCM output, showed no change in their differential LCM activity after training.

In the previous paper we reported that differential LCM activity could be quantitativcly related to the magnitude and direction of the horizontal head-waving response. (Cook and $\mathrm{Ca}$ rew, 1989a). The significant change in differential LCM output following contingent training described in the present study should allow us to estimate the expected change in the magnitude of the behavioral head-waving response by assessing the magnitude of the change in LCM activity. Following contingent training of the LCMs, their mean differential change in the firing rate in the present study was 3.25 spikes/sec. Using linear-regression equations derived from the data in the previous paper, we estimated that this change in firing rate should accompany a mean shift in the animal's head position of approximately $10^{\circ}$. However, the magnitude of horizontal head-waving behavior required to initiate or terminate reinforcement in our behavioral experiments is at least $13.5^{\circ}$ (Cook and Carew, 1986). Thus, even though the LCMs were contingently modified, the animals' expected head-waving response commensurate with this amount of LCM change would not be sufficient to give rise to reinforcement during behavioral conditioning. Consistent with this line of reasoning, behavioral scores, taken blind with respect to LCM activity during baseline and test periods, revealed no evidence of behavioral operant conditioning in our present experiments. These results suggest, first, that other muscles, in addition to the LCMs, may play an important role in head-waving and its operant modification (see Cook and Carew, 1989a, for discussion) and, second, that the LCMs are an extremely sensitive system in which to assess the modification of response output by contingent reinforcement, perhaps even more sensitive than the behavioral response itself.

\section{Implication for a cellular analysis of operant conditioning}

In broad conceptual terms, for operant modification of a response to take place, at least 2 cellular events must occur: (1) the organism must have some means of monitoring the operant behavioral response (i.e., via reafferent feedback and/or central efference copy), and (2) the occurrence of reinforcement must be registered and then somehow interact with the cellular registration of the behavioral response. The neuronal sites where such interactions occur are prime candidate loci for the detection and storage of associations between the occurrence of the operant response and subsequent reinforcement. Thus, identification and characterization of the operant response and reinforcement pathways are critical steps in the analysis of the cellular basis of operant conditioning. By establishing that the EMG activity of the LCMs can be modified by contingent reinforcement, we have identified a restricted motor output pathway that retains the essential features of operant conditioning of headwaving. This restricted system now permits examination of a number of important questions in the operant response pathway. For example, does the contingent modification of LCM activity require feedback from the LCMs themselves? Can direct activation of the LCMs (i.e., by means of electrical stimulation of the muscles or peripheral nerves to the muscles), paired with reinforcement, give rise to operant modification of LCM activity? Finally, can the activity of individual motor neurons in- nervating the LCMs also be contingently modified? We have recently obtained preliminary evidence that bears on this last issue. Specifically, we have found that individual motor neurons that innervate the neck and whose activity is modulated by peripheral photic input (Cook and Carew, 1989b) can be contingently modified to decrease their tonic firing rate significantly by punishing increases in firing rate with aversive, whole-body illumination. Yoked control cclls show no decrcasc in firing ratc following training with noncontingent reinforcement (Cook and Carew, 1988). This modification of individual motor neurons, by contingent reinforcement, provides a useful starting point from which to examine possible central cellular sites of plasticity underlying operant conditioning of head-waving in Aplysia.

\section{References}

Abramson, C. I., and R. D. Feinman (1987) Operant conditioning of eye elevation in the green crab. Behav. Neural Biol. 48: 259-277.

Alkon, D. L. (1987) Memory Traces in the Brain, Cambridge U. P., Cambridge, England.

Bablanian, G. M., K. R. Wciss, and I. Kupfermann (1987) Motor control of the appetitive phase of feeding behavior in Aplysia. Behav. Neural Biol. 48: 394-407.

Brace, R. C. (1977a) The functional anatomy of the mantle complex and columellar muscle of tectibranch molluscs (Gastropoda: Opisthobranchia), and its bearing on the evolution of opisthobranch organization. Phil. Trans. R. Soc. London [Biol.] 277: 1-56.

Brace, R. C. (1977b) Shell attachment and associated musculature in the notaspidea and anaspidea (Gastropoda: Opisthobranchia). Trans. Zool. Soc. London 34: 27-43.

Byrne, J. H. (1987) Cellular analysis of associative learning. Physiol. Rev. 67: 329-439.

Carew, T. J., and C. L. Sahley (1986) Invertebrate learning and memory: From behavior to molecules. Annu. Rev. Neurosci. 9: 435-487.

Carew, T. J., R. D. Hawkins, and E. R. Kandel (1983) Differential classical conditioning of a defensive withdrawal reflex in Aplysia californica. Science 219: 397-400.

Cobbs, J. S., and H. M. Pinsker (1982a) Role of bag cells in egg deposition of Aplysia brasiliana. I: Comparison of normal and elicited behaviors. J. Comp. Physiol. 147: 523-536.

Cobbs, J. S., and H. M. Pinsker (1982b) Role of bag cells in egg deposition of Aplysia brasiliana. II: Contribution of egg movement of elicited behaviors. J. Comp. Physiol. 147: 537-546.

Cook, D. G., and T. J. Carew (1986) Operant conditioning of headwaving in Aplysia. Proc. Natl. Acad. Sci. USA 83: 1120-1124.

Cook, D. G., and T. J. Carew (1988) Operant conditioning of identified neck muscles and individual motor neurons in Aplysia. Soc. Neurosci. Abstr. 14: 607.

Cook, D. G., and T. J. Carew (1989a) Operant conditioning of headwaving in Aplysia. I. Identified muscles involved in the operant response. J. Neurosci. 9: 3097-3106.

Cook, D. G., and T. J. Carew (1989b) Operant conditioning of headwaving in Aplysia. III. Cellular analysis of possible reinforcement pathways. J. Neurosci. 9: 3115-3122.

Crow, T. (1985a) Conditioned modification of phototactic behavior in Hermissenda. I. Analysis of light intensity. J. Neurosci. 5: 209214.

Crow, T. (1985b) Conditioned modification of phototactic behavior in Hermissenda. II. Differential adaptation of B photoreceptors. J. Neurosci. 5: 215-223.

Crow, T. J. (1988) Cellular and molecular analysis of associative behavioral changes in Hermissenda. Trends Neurosci. 11: 136-142.

Downey, P., and B. Jahan-Parwar (1972) Cooling as rcinforcing stimulus in Aplysia. Am. Zool. 12: 507-512.

Farley, J., and D. L. Alkon (1982) Associative neural and behavioral change in Hermissenda: Consequences of nervous system orientation for light- and pairing-specificity. J: Neurophysiol. 48: 785-807.

Fredman, S. M., and B. Jahan-Parwar (1983) Command neurons for locomotion of Aplysia. J. Neurophysiol. 49: 1092-1117.

Hawkins, R. D., T. W. Abrams, T. J. Carew, and E. R. Kandel (1983) A cellular mechanism of classical conditioning in Aplysia: Activitydependent amplification of presynaptic facilitation. Science 219:400405 . 
Hawkins, R. D., G. A. Clark, and E. R. Kandel (1985) Operant conditioning and differential classical conditioning of gill withdrawal in Aplysia. Soc. Neurosci. Abstr. 11: 796.

Hawkins, R. D., G. A. Clark, and E. R. Kandel (1987) Cell biological studies of learning in simple vertebrate and invertebrate systems. In Handbook of Physiology, Sect. I, Higher Functions of the Nervous System, Vol. 6, F. Plum, ed., pp. 25-83, American Physiological Society, Bethesda, MD.

Hening, W. A., E. T. Walters, T. J. Carew, and E. R. Kandel (1979) Motoneuronal control of locomotion in Aplysia. Brain Res. 179: 231253.

Hoyle, G. (1982a) Cellular basis of operant-conditioning of leg position. In Conditioning: Representation of Involved Neural Functions, C. D. Woody, ed., pp. 197-211, Plenum, New York.

Hoyle, G. (1982b) Pacemaker change in learning paradigm. In Cellular Pacemakers, D. Carpenter, ed., pp. 3-25, Wiley, New York.

Kuenzi, F. M., and T. J. Carew (1988) A biomechanical analysis of the head-waving response in Aplysia. Soc. Neurosci. Abstr. 14:1000.

Kupfermann, I. (1974) Feeding behavior in Aplysia: A simple system for the study of motivation. Behav. Biol. 10:1-26.

Kupfermann, I., and T. J. Carew (1974) Behavioral patterns of Aplysia californica in its natural environment. Behav. Biol. 12: 317-337.

Lee, R. M. (1969) Aplysia behavior: Effects of contingent water-level variation. Commun. Behav. Biol., Pt. A 4: 157-164.

Lee, R. M. (1970) Aplysia behavior: Operant-response differentiation. Proc. Annu. Conv. Am. Psychol. Assoc. 78: 249-250.

Mackintosh, N. J. (1974) The Psychology of Animal Learning, Academic, London.

Pinsker, H. M., and L. B. Eberly (1982) Whole-nerve cuff electrodes in neuroethological studies. J. Electrophysiol. Tech. 8: 88-101.

Preston, R. J., and R. M. Lee (1973) Feeding behavior in Aplysia californica: Role of chemical and tactile stimuli. J. Comp. Physiol. 82: 368-381.

Rescorla, R. A. (1988) Behavioral studies of Pavlovian conditioning. Annu. Rev. Neurosci. 11: 329-352.

Reynolds, G. S. (1968) Primer of Operant Conditioning, Scott Foresman, Glenview, IL.

Schwarz, M., and A. J. Susswein (1986) Identification of the neural pathway for reinforcement of feeding when Aplysia learn that food is inedible. J. Neurosci. 6: 1528-1536.

Stein, L., and J. D. Belluzzi (1988) Operant conditioning of individual neurons. In Quantitative Analysis of Behavior: Biological Determinants of Reinforcement, Vol. 7, M. Connors, R. Church, J. Stellar, and Wagner, eds., pp. 249-264, Erlbaum, Hillsdale, NJ.

Susswein, A. J., and J. H. Byrne (1988) Identification and characterization of neurons initiating patterned neural activity in the buccal ganglion of Aplysia. J. Neurosci. 8: 2049-2061.

Susswein, A. J., and M. Schwarz (1983) A learned change of response to inedible food in Aplysia. Behav. Neural Biol. 39: 1-6.

Susswein, A. J., M. Schwarz, and E. Feldman (1986) Learned changes of feeding behavior in Aplysia in response to edible and inedible foods. J. Neurosci. 6: 1513-1527.

Walters, E. T., and J. H. Byrne (1983a) Associative conditioning of single sensory neurons suggests a cellular mechanism for learning. Science 219: 405-408.

Walters, E. T., and J. H. Byrne (1983b) Slow depolarization produced by associative conditioning of Aplysia sensory neurons may enhance $\mathrm{Ca}^{++}$entry. Brain Res. 280: 165-168.

Woolacott, M. H., and G. Hoyle (1977) Neural events underlying learning in insects: Changes in pacemaker. Proc. R. Soc. London Ser B 195: 395-415. 